RESEARCH HIGHLIGHTS

\title{
Sizing up the fly
}

The final size of an organism depends on both its rate and duration of growth - but how are these variables controlled and coordinated? A recent flurry of studies in the fruitfly has pieced together some important parts of this puzzle.

In Drosophila melanogaster, growth takes place over three larval stages, during which enough food needs to be consumed to survive the rest of development. Larvae at the third stage must reach a critical size to undergo pupariation, a process that marks the transition to metamorphosis and is triggered by the release of the steroid hormone ecdysone from the prothoracic gland (PG).

Caldwell and colleagues showed that expressing constitutively active components of the RAS signalling pathway in the PG led to a reduction in final body size. By contrast, suppressing this pathway resulted in an extended duration of the larval stages and an increase in body size. Monitoring the expression of ecdysone-responsive transgenes revealed that activated RAS in the PG leads to premature ecdysone release. So, the RAS pathway regulates body size by regulating ecdysone production, and therefore the duration of growth.

However, the same study indicated that RAS signalling is not the only pathway that regulates body size. Expression of activated phosphatidylinositol 3-kinase (PI3K) was found to decrease body size independently of RAS activity. The authors postulated that this effect of
PI3K is mediated through its ability to increase the size of PG cells, which would increase ecdysone production and terminate larval growth prematurely. This is consistent with a study by Mirth and colleagues, which showed that the PG assesses when the larva has reached the critical size and subsequently triggers ecdysone release. Importantly, $\mathrm{PI} 3 \mathrm{~K}$ is a downstream effector of the insulin signalling pathway, which is known to couple fly body size to the availability of nutrients, and these findings provide new insights into the underlying mechanisms.

A paper by Colombani and colleagues reports similar effects on body size after genetic manipulation of PI3K in the PG. Interestingly, this study also establishes a new role for ecdysone in regulating animal growth rate, through a general repression of insulin signalling in peripheral tissues. So, insulin and ecdysone seem to antagonize each other's effects on growth rate, and thereby regulate body size.

Finally, Shingleton and colleagues investigated whether insulin signalling exerts the same effect on body size at all larval stages. Using a temperature-sensitive mutation in the insulin receptor (Inr) gene they showed that disrupting this pathway before the critical size is reached extends total development time, but has no effect on the final size of the fly. The opposite was true when INR signalling was blocked after the critical size had been attained.

These studies represent impor- tant steps in understanding how body size is regulated in the fly. They might also have implications for understanding the coordination of nutrient availability and developmental transitions in mammals, where steroid hormones and insulin signalling also have key roles in regulating growth.

Louisa Flintoft

6) References and links ORIGINAL RESEARCh PAPERS Caldwell, P. E., Walkiewicz, M. \& Stern, M. Ras activity in the Drosophila prothoracic gland regulates body size and developmental rate via ecdysone release. Curr. Biol. 20 September 2005 (doi:10.1016/ j.cub.2005.09.011) | Mirth, C., Truman, J. W. \& Riddiford, L. M. The role of the prothoracic gland in determining critical weight for metamorphosis in Drosophila melanogaster. Curr. Biol. 20 September 2005 (doi:10.1016/ j.cub.2005.09.017) | Colombani, J. et al. Antagonistic actions of ecdysone and insulins determine final size in Drosophila. Science 22 September 2005 (doi:10.1126/ science.1119432) | Shingleton, A. W. et al. The temporal requirements for insulin signaling during development in Drosophila. PLoS Biol. 3, e289 (2005)

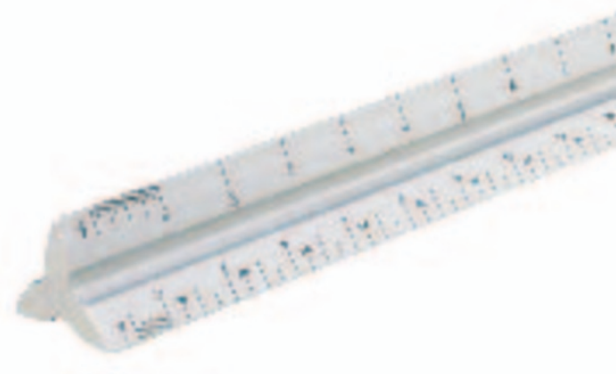

